CMAJ FACT SHEET

\section{Rabies risk among travellers}

\section{How do you get rabies?}

Rabies is a preventable, deadly disease transmitted through contact with an infected warm-blooded animal. Most often people are bitten, but in some cases they may have a non-bite exposure to the saliva of a rabid animal, for example through licks on broken skin or mucous membranes in the eyes, nose or mouth, or scratches that break the skin. Although extremely rare, rabies may occur after exposure to aerosolized rabies virus in a cave filled with infected bats.

In developing countries, dogs remain the main source of rabies, with monkeys the second most common source. In most developed countries, skunks, raccoons, bats and foxes are the main source.

\section{Are travellers at risk for rabies?}

Although rabies is rare among international travellers, many rabies-related deaths in the developed world have involved people who were infected while travelling. There has been only I travelrelated Canadian case of rabies in the last 70 years.

\section{What countries pose a high risk for rabies?}

Rabies occurs worldwide with few exceptions, including Antarctica, New Zealand, Japan, Taiwan, parts of Europe (e.g., Sweden and Norway), some Caribbean islands and Hawaii. The highest incidence of rabies continues to be in Africa and Asia, particularly India. Most deaths from rabies occur in India and Southeast Asia, Africa and Latin America. Thailand has an estimated Io million stray dogs, with I in ro dogs in Bangkok estimated to be infected with rabies.

\section{Who is at highest risk?}

People travelling to rural areas or areas heavily populated with stray dogs in rabies-endemic countries are at highest risk. Children (boys more than girls) are 4 times as likely as adults to get rabies because they are more likely to be bitten and less likely to report it. People who participate in spelunking and other cave-related activities are at high risk, as are those who have unprotected outdoor, evening or nighttime exposures in rural areas, such as those biking, camping or doing volunteer work.

\section{What is the risk of rabies from a dog bite when travelling?}

Dog bites are a common occurrence among tourists. In one study, 13 dog bites per 1000 people visiting Thailand were reported. Many dogs in foreign countries are not vaccinated against rabies. The risk of infection following an exposure to a rabid ani$\mathrm{mal}$ is about $15 \%$, but it varies (from $0.1 \%$ to $60 \%$ ) depending on the exposure factors of the bite. These factors include the number of bites, the depth of the bites and the stage of illness in the infected animal. Injury to the upper body or head poses the greatest risk of transmission.

\section{How can you avoid rabies?}

The main way to avoid rabies is to avoid contact with wild or stray animals. All stray dogs in foreign countries should be presumed to have rabies, even if the animal appears friendly. All contact with bats should be avoided, and bats should never be handled. In addition, monkeys should not be handled, and food should not be carried when visiting areas where monkeys congregate.

\section{What do you do if you are bitten while travelling?}

If bitten, scratched, or licked on mucous membranes or an open wound by any animal in a foreign country, immediately wash the wound thoroughly and vigorously with soap and water, and with a povidone-iodine compound if available. Seek medical treatment immediately to receive postexposure vaccination ( 5 doses over 30 days for those who have not previously received preexposure vaccination) and human rabies immune globulin (a single dose within 7 days of the first vaccine dose if not previously vaccinated). The incuba- tion period for rabies is usually 20-60 days, but it may be prolonged (more than a year); therefore, it is never too late to receive treatment before symptoms develop. A special consideration is bat bites. Bats have small teeth and can inflict bites or scratches without leaving behind a noticeable wound. Therefore, if a bat is found in a room where a person has been sleeping, that person should be considered to have been exposed to rabies.

\section{Who should be vaccinated before international travel?}

Rabies vaccine substantially reduces the risk of infection when given before or after potential exposure. Those who receive the vaccine before travel will still require 2 additional doses after a potential rabies exposure. However, they will not require human rabies immune globulin, a product often unavailable in developing countries. This is the main reason for the recommendation for pre-travel vaccination. Because of the high cost of the rabies vaccine, pre-exposure vaccination is recommended only for people who are travelling to rabies-endemic areas (especially for prolonged stays), are likely to come into contact with wild or stray animals and will not have rapid access (within 3 days) to medical care. Preexposure vaccination is also recommended for people travelling to countries where postexposure prophylaxis is not available or is unsafe. The Public Health Agency of Canada strongly recommends that travellers obtain an individual risk assessment from a physician or travel medicine clinic before departure (www.travelhealth.gc.ca).

\section{Melanie Di Quinzio MD MSc \\ Division of Internal Medicine University of Ottawa \\ Anne McCarthy MD MSc \\ Division of Infectious Diseases \\ Ottawa Hospital \\ Ottawa, Ont.}

This article has been peer reviewed.

Competing interests: None declared.

Une version française de cet article est disponible à l'adresse www.cmaj.ca/cgi /content/full/I78/5/567/DCI 